\title{
Repräsentation vor Ort: Selbstverständnis und Verhalten von Bundestagsabgeordneten bei der Wahlkreisarbeit*
}

\author{
Sven T. Siefken
}

Wer die Arbeit von Abgeordneten untersucht, blickt meist in das Parlament hinein: Dann stehen Plenardebatten, Gesetzgebungsprozesse und die fachlich hochspezialisierte Tätigkeit in Ausschüssen und Fraktionen im Mittelpunkt des Interesses. Dies gilt für die allgemeine öffentliche Wahrnehmung ebenso wie für die journalistische Betrachtung und auch - mit wenigen Ausnahmen - für die wissenschaftliche Untersuchung. ${ }^{1}$ Richard F. Fenno hat für die USA festgehalten, was auch für Deutschland zutrifft: „The home connections of our elected politicians are understudied and underreported elements of our representational system of government. "2 Dies sind höchst wichtige Elemente, denn die Praxis sieht so aus: Mitglieder des Deutschen Bundestages verbringen einen Gutteil ihrer Zeit mit der Arbeit in ihren Wahlkreisen: Eine Größenordnung von durchschnittlich 40 Prozent erscheint realistisch. ${ }^{3}$

Die zeitliche Bedeutung der Arbeit vor Ort ist nicht nur als empirische Erkenntnis interessant, sondern theoretisch von höchster Relevanz, denn in einer repräsentativen Demokratie gilt - in den Worten von Hanna F. Pitkin: „Representing ... means acting in the interest of the represented in a manner that is responsive to them. " 4 Zur Aufnahme der Interessen, zur Vermittlung des Handelns und mithin zur Herstellung von Responsivität

* Für die Möglichkeit, sie in ihren Wahlkreisen begleiten zu dürfen, dankt das CITREP-Team den beteiligten Abgeordneten des Deutschen Bundestages, für die Unterstützung bei der Datenerhebung den Doktoranden und Studierenden an den Universitäten Halle und Stuttgart, die als Beobachter eingesetzt waren. Für die Recherche, Interviewtranskription, Kodierung und Vorbereitung der Datenanalyse gilt ein besonderer Dank Şervan Deniz und Benjamin Greiner sowie Julia Schubert, Marcus Wittig und Florian Hüfner.

1 Vgl. für Deutschland die Studien von Werner J. Patzelt, Abgeordnete und Repräsentation: Amtsverständnis und Wahlkreisarbeit, Passau 1993; ders., Abgeordnete und ihr Beruf. Interviews Umfragen - Analysen, Berlin 1995; ders., Deutschlands Abgeordnete: Profil eines Berufsstands, der weit besser ist als sein Ruf, in: ZParl, 27. Jg. (1996), H. 3, S. 462 - 502; ders. / Karin Algasinger, Abgehobene Abgeordnete? Die gesellschaftliche Vernetzung der deutschen Volksvertreter, in: ZParl, 32. Jg. (2001), H. 3, S. 503 - 527; für die Vereinigten Staaten von Amerika Richard F. Fenno, Home Style. House Members in their Districts, Boston 1978, im Überblick zu jener und weiterer Studien: ders., Congressional Travels: Places, Connections, and Authenticity, New York 2007.

2 Ders., Congressional Travels, a.a.O. (Fn. 1), S. 41.

3 Dies ist ein Schätzwert auf Basis von Gesprächen mit Parlamentariern. Detaillierte Arbeitszeitanalysen existieren für Abgeordnete nicht; in Abhängigkeit von Führungsämtern in Fraktion und Regierung ist zudem von erheblichen Schwankungen auszugehen. Frühere Zeitbudgeterhebungen haben die Verwendung der Arbeitszeit von Abgeordneten in Parlaments- und Sitzungswochen aufgabenbezogen unterteilt, nicht aber zusammengeführt, vgl. Paul Kevenhörster / WulfSchönbohm, Zur Arbeits- und Zeitökonomie von Bundestagsabgeordneten, in: ZParl, 4. Jg. (1973), H. 1, S. 18 - 37; Dietrich Herzog / Hilke Rebenstorf / Camilla Werner / Bernhard Weßels (Hrsg.), Abgeordnete und Bürger. Ergebnisse einer Befragung der Mitglieder des 11. Deutschen Bundestages und der Bevölkerung, Opladen 1990, S. 83 ff.

4 Hanna F. Pitkin, The Concept of Representation, Berkeley 1967, S. 209. 
bedarf es der Wahlkreisarbeit. In Realisierung dieser grundsätzlichen Erfordernis sind gemäß der Logik des parlamentarischen Regierungssystems der Bundesrepublik die Wahlkreise funktional in vierfacher Hinsicht von besonderer Bedeutung:

- Erstens sind sie die organisatorische Einheit für die Besetzung der Direktmandate im Rahmen der Bundestagswahlen (Wahl).

- Zweitens, und zeitlich davor, stellen die Parteien in den Wahlkreisen ihre Kandidaten für die Direktmandate im Rahmen von Wahlkreiskonferenzen auf (Nominierung).

- Drittens sind die Wahlkreise eine Quelle von Fach- wie Alltagswissen für die Abgeordneten, die im Rahmen der Parlamentsarbeit genutzt werden kann (Information).

- Viertens sind sie ein Ort politischer Kommunikation, in der Repräsentanten und Repräsentierte sich miteinander austauschen (Kommunikation).

Aus der theoretischen Erörterung dieser vier funktionalen Perspektiven ergeben sich Erwartungen an die Wahrnehmung und Ausgestaltung der Wahlkreisarbeit durch die Abgeordneten. Sie werden im Folgenden hergeleitet und jeweils mit den empirischen Erkenntnissen aus bereits vorliegenden wissenschaftlichen Arbeiten sowie den Ergebnissen ausführlicher Beobachtungen und Interviews bei 67 Abgeordneten des Deutschen Bundestages in den Jahren 2011 und 2012 überprüft.

\section{Datengrundlage: Projekt "Citizens and Representatives in France and Germany"}

Die verwendeten Daten stammen aus dem Forschungsprojekt „Citizens and Representatives in France and Germany" (CITREP), das in den Jahren 2010 bis 2013 unter Leitung von Oscar W. Gabriel (Universität Stuttgart), Suzanne S. Schüttemeyer (Martin-Luther-Universität Halle-Wittenberg) und Eric Kerrouche (Sciences Po, Bordeaux) durchgeführt wurde. ${ }^{5}$ Im Rahmen der Untersuchungen wurden in Deutschland 64 Abgeordnete des Deutschen Bundestages zwischen Februar 2011 und Mai 2012 in der Regel für jeweils drei Tage bei ihrer Wahlkreisarbeit begleitet. Richard F. Fenno hat die teilnehmende Beobachtung als Methode für die Abgeordnetenforschung in den USA etabliert und über Jahrzehnte bei Mitgliedern des U.S.-Kongresses immer wieder angewandt. ${ }^{6}$ Während Fenno über längere Zeiträume als alleiniger Beobachter unterwegs war, Daten in ihrer realen Umgebung zunächst breit erhoben und später induktiv analysiert hat, konnte für CITREP bereits auf vorhandene Forschungserkenntnisse aufgebaut werden. So kam eine stärker strukturierte Erhebung zum Einsatz, in der jede begleitete Veranstaltung mit Hilfe eines einheitlichen Erhebungsbogens aufgenommen wurde, der nominal skalierte Daten (zum Beispiel „Waren Medienvertreter anwesend? Ja/Nein“), überwiegend ordinale Einschätzungen auf Fünfer-Skalen („Wie häufig wurden folgenden Politikfelder thematisiert? Sehr häufig, häufig, mittel, selten, sehr selten.“) und einige metrische Informationen (zum Beispiel „Wie viele Teilnehmer hatte die

5 Das Projekt wurde gefördert durch die Deutsche Forschungsgemeinschaft und die Agence Nationale de la Recherche in Frankreich, vgl. www.citrep.eu; eine umfassende Vorstellung erster Ergebnisse und Diskussion mit Abgeordneten fand statt im Rahmen der internationalen Konferenz „Repräsentation im Wahlkreis - Bevölkerung und Abgeordnete in Deutschland und Frankreich“, veranstaltet von der Deutschen Vereinigung für Parlamentsfragen in Berlin am 7./8. Dezember 2012 .

6 Vgl. in der Gesamtschau seiner Forschungsarbeiten Richard F. Fenno, Congressional Travels, a.a.O. (Fn. 1). 
Veranstaltung?“”, „Wie lange dauerte sie?“) enthielt. In offenen Fragen und vor allem in den frei zu formulierenden Protokollen konnten die Beobachter zudem ihre persönlichen Eindrücke schildern, aussagekräftige Zitate aufnehmen oder Situationen detailliert darstellen. So wurden insgesamt 618 Veranstaltungen mit einer Nettodauer von 969 Stunden erfasst. Die Beobachter hatten persönlich Zugang zu 86 Prozent dieser Veranstaltungen; Grunddaten für die anderen wurden gemäß Auskunft der Teilnehmer bereitgestellt. Für die Verwendung der aus den Beobachtungen erhobenen Informationen wurde den Abgeordneten Anonymität zugesichert.

Zusätzlich wurde mit den Abgeordneten, meist am dritten Tag der Begleitung, ein ausführliches leitfadengestütztes Interview geführt. 45 Fragen behandelten Themen wie parlamentarische Repräsentation allgemein, Organisation und Schwerpunktsetzung bei der Wahlkreisarbeit, die Erwartungshaltung von Bürgern sowie die begleiteten Veranstaltungen. Dieses Erhebungsdesign, in dem Interviews zum Abschluss einer mehrtägigen Begleitung geführt wurden, profitierte davon, dass sich zwischen Beobachter und Abgeordneten häufig ein Vertrauensverhältnis entwickelt hatte und somit auch die Interviewführung deutlich erleichtert wurde. Bei drei Abgeordneten ließen sich aus terminlichen Gründen innerhalb des Untersuchungszeitraumes keine Beobachtungen vor Ort realisieren, so dass mit ihnen nur die Interviews geführt wurden; die Gesamtzahl der Interviews liegt daher bei 67. Sie wurden alle vollständig transkribiert und den Abgeordneten zur Autorisierung vorgelegt, so dass sie auch namentlich zitiert werden können. Die Kodierung und Auswertung erfolgte mit Hilfe der Software MaxQDA.

Insgesamt sind über ein Zehntel (elf Prozent) der Abgeordneten des 17. Deutschen Bundestages Teil der Stichprobe. Sie kam nicht durch ein Zufallsverfahren zustande, sondern mittels eines theoretischen Sampling, das 19 Kriterien der Person und des Wahlkreises berücksichtigte, die aus theoretischer Sicht das Repräsentationsverhalten beeinflussen können und vorab festgelegt wurden. Dazu gehören für die Abgeordneten das Alter, die Seniorität, die Parteizugehörigkeit und der Mandatstyp (direkt oder über Liste gewählt) und für die Wahlkreise der Urbanisierungsgrad, die Arbeitslosenquote und die Stärke der Partei vor Ort („Stimmenhochburg“ oder „Diaspora“). Die Verteilung der Auswahlkriterien in der Stichprobe entspricht jener im Deutschen Bundestag der 17. Wahlperiode. So sind auf Basis dieser systematischen Auswahl zwar keine inferenzstatistisch abgesicherten Generalisierungen von der Stichprobe auf die Grundgesamtheit aller Abgeordneten möglich, doch liefern die erhobenen Daten Befunde, die wohl für die allermeisten Abgeordneten im Bundestag zutreffen.

\section{Die Bedeutung der Wahlkreise im politischen System Deutschlands}

\subsection{Ort der Wahl - und des Wahlkampfes für (fast) alle Abgeordneten}

Gemäß dem Bundeswahlgesetz ist Deutschland in 299 Wahlkreise unterteilt. In jedem dieser Wahlkreise gewinnt der Abgeordnete das so genannte Direktmandat, der die einfache Mehrheit der abgegebenen Erststimmen erhält. Die gesamten Parlamentsmandate werden gemäß der Verteilung der Zweitstimmen verteilt, wobei zunächst die schon vergebenen Direktmandate abgezogen werden. Erhält eine Partei innerhalb eines Landes mehr Direktmandate als ihr nach den Zweitstimmen zustehen würden, entstehen die so genannten 
Überhangmandate. Bei der Bundestagswahl 2013 kommt erstmals ein Ausgleichsverfahren zum Einsatz, um sicherzustellen, dass auch nach der Zuteilung von Überhangmandaten noch das Verhältnis der Zweitstimmen auf Bundesebene gewahrt ist. Insofern war - und bleibt - das Wahlrecht der Bundesrepublik eine Verhältniswahl mit einer wichtigen Komponente der Personalisierung. Diese Komponente führt zur zentralen Bedeutung der Wahlkreise, denn wer dort die relative Mehrheit gewinnt, zieht auf jeden Fall in das Parlament ein. Regelmäßig sind es vor allem die Kandidaten der großen Parteien SPD und CDU, der regional starken CSU in Bayern und der Linkspartei im Osten Deutschlands, die Direktmandate gewinnen. In Wahlkreisen mit besonderer Zusammensetzung haben aber auch schon Abgeordnete der kleinen Parteien gewonnen, so Hans-Christian Ströbele (Bündnis 90/ Die Grünen) seit 2002 im Wahlkreis 84 (Berlin-Friedrichshain-Kreuzberg-Prenzlauer Berg Ost). Insofern gibt es für Abgeordnete insbesondere der großen Parteien klare Anreize, sich um ihren Wahlkreis und die potenziellen Wähler dort besonders zu kümmern. Die Wahlkreise sind folgerichtig ein wichtiger Ort der Wahlkampagnen, für die die Kandidaten auf freiwillige Unterstützer angewiesen sind.7 Daher ist davon auszugehen, dass auch eine deutliche Pflege der Parteibasis erfolgt.

Aus organisatorischen Gründen wird Abgeordneten von den Fraktionen häufig ein größerer Betreuungsbereich zugewiesen, um auch jene Wahlkreise abzudecken, in denen die eigenen Kandidaten nicht in den Bundestag eingezogen sind: 55 Prozent gaben an, dass sie einen solchen zusätzlichen Bereich betreuen, und zwar sowohl in großen wie kleinen Fraktionen. Im Gespräch betonen sie dann aber, „das emotionale Verhältnis und auch die Intensität der Betreuung “ des eigenen Wahlkreises sei deutlich intensiver als für den Betreuungsbereich (Johannes Vogel, MdB, FDP) oder: „Natürlich ist das mein Herzenswahlkreis, aber [es] gehört auf jeden Fall der gesamte ostfriesische Teil zu dem Bereich, den ich zu betreuen habe." (Hans-Michael Goldmann, MdB, FDP)

$\mathrm{Zu}$ beachten ist also, dass der Begriff Wahlkreisarbeit nicht exklusiv auf die Tätigkeit von direkt gewählten Abgeordneten innerhalb der Wahlkreisgrenzen gemäß dem Bundeswahlgesetz beschränkt werden kann: Auch Listenkandidaten leisten Wahlkreisarbeit, und sie findet auch außerhalb des eigenen Wahlkreises statt. Die Definition „Wahlkreis bezeichnet einen räumlich genau festgelegten Teil eines politischen Gemeinwesens "8 mag also aus wahlrechtlicher Sicht zutreffen, für die Untersuchung von Repräsentation in der politischen Praxis ist sie aber zu eng. Allgemein kann Wahlkreisarbeit besser definiert werden als die Tätigkeit von Abgeordneten vor Ort jenseits der unmittelbaren Spezialisierung der Parlamentstätigkeit.

Auf die offene Frage, was die wichtigsten Dinge sind, die sie im Wahlkreis tun, gaben die begleiteten Abgeordneten naturgemäß vielfältig Auskunft. Ihre Antworten lassen sich in sechs Gruppen zusammenfassen (vgl. Abbildung 1): Deutlich im Vordergrund steht die Kontaktpflege und der Austausch (genannt von 38 Abgeordneten, das heißt 57 Prozent) sowie die Informationsaufnahme (48 Prozent), wie ein Abgeordneter erläutert:

7 Vgl. Thomas Bosch, „Hinten sind die Enten fett“. Der Bundestagswahlkampf der SPD und die Mobilisierung der eigenen Mitglieder, in: Christina Holtz-Bacha (Hrsg.), Die Massenmedien im Wahlkampf. Die Bundestagswahl 2005, Wiesbaden 2006, S. 32 - 79.

8 Dieter Nohlen, Wahlkreis, in: Klaus Schubert / Martina Klein (Hrsg.), Das Politiklexikon. Begriffe, Fakten, Zusammenhänge, Bonn 2011, http://www.bpb.de/nachschlagen/lexika/politiklexikon/18457/wahlkreis (Abruf am 31. August 2013). 


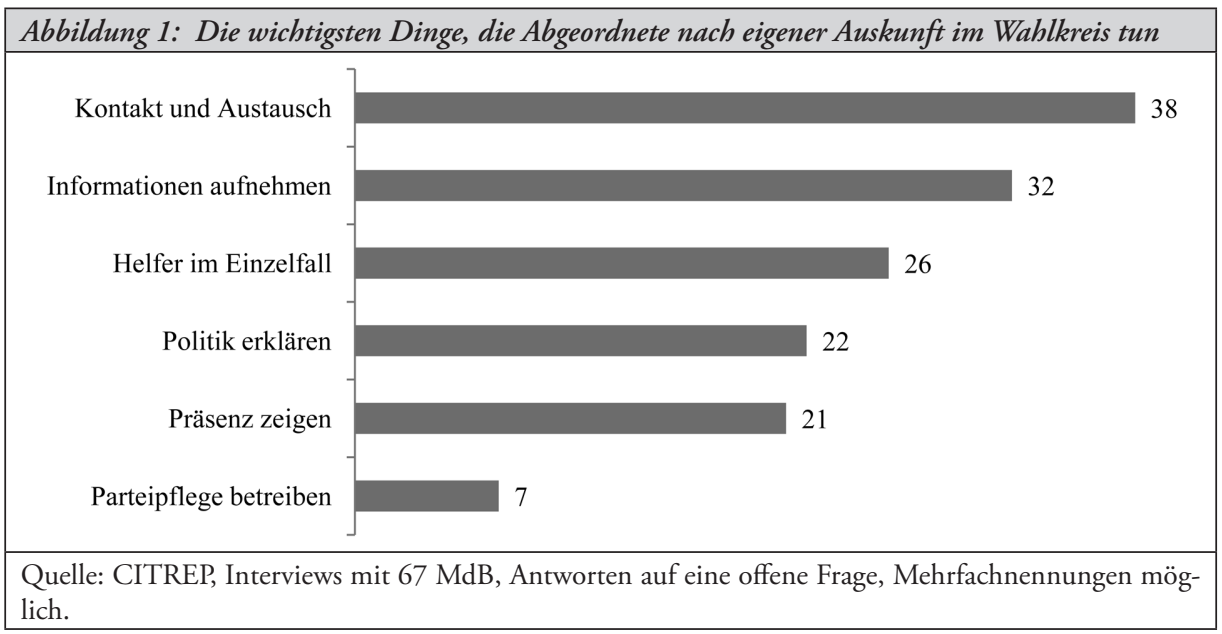

„[Ich] versuche möglichst viele Eindrücke aus der Lebenswirklichkeit der Menschen - hier vor Ort - mitzunehmen und nutze dies als Grundlage für meine politischen Entscheidungen und meine politische Arbeit in Berlin." (Josip Juratovic, MdB, SPD)

Überraschend stark ist die Betonung der Hilfestellung im Einzelfall (39 Prozent), die von der Unterstützung beim Ausfüllen von Anträgen bis zur Rechtsberatung reicht, was in den folgenden Aussagen beispielhaft illustriert wird:

„Ansonsten werde ich auf Anfrage aktiv. Wenn es irgendwo Probleme, Sorgen und Nöte im Wahlkreis gibt, versuche ich mich darum zu kümmern." (Stefan Liebich, MdB, Die Linke)

„Ich bin für die Menschen hier zuständig, in allen ihren Facetten, vor allem mit einer bundespolitischen Betrachtungsweise, aber auch für viele konkrete Lebenshilfen, sobald sie gewünscht werden." (Dieter Wiefelspütz, MdB, SPD)

Im föderalen System der Bundesrepublik wird ein Großteil der Verwaltungsaufgaben durch Länder und Kommunen wahrgenommen, und so hätten Abgeordnete eigentlich leicht die Möglichkeit, entsprechende Bitten um Hilfe an die „zuständige“ Stelle zu verweisen. Doch dies tun sie nicht, sondern kümmern sich häufig selbst darum: Sie rufen Anwälte an, helfen beim Ausfüllen von Sozialversicherungsanträgen, unterstützen bei Terminen im Ausländeramt oder bei der Beantragung von Fördergeldern. Dieser Themenbereich des individuellen „Casework“ bedarf näherer Untersuchung, zumal vergleichende Studien vor dessen Inflation und einem mangelnden strategischen Zugang der Parlamente dazu warnen. ${ }^{9}$

Allerdings stellt sich die Hilfe im Einzelfall bei den Beobachtungen anders dar als in der Selbstauskunft: Nur in zehn Prozent der Veranstaltungen nahm sie einen großen oder sehr großen Raum ein (vgl. Abbildung 2), und der Zeitanteil der beobachteten Bürgersprechstunden lag lediglich bei sieben Prozent (vgl. Abbildung 4). Dies stimmt überein mit dem Ergebnis einer experimentellen Untersuchung, wonach Abgeordnete dieser so genannten „Dienstleistungs-Responsivität“ („service responsiveness“) ${ }^{10}$ wohl normativ einen hohen Stellenwert

9 Vgl. IPU / UNDP, Global Parliamentary Report. The Changing Nature of Parliamentary Representation, Geneva / New York 2012, S. 7.

10 Vgl. Heinz Eulau / Peter D. Karps, The Puzzle of Representation: Specifying Components of Responsiveness, in: Legislative Studies Quarterly, 2. Jg. (1977), H. 3, S. 233 - 254, S. 245. 
zuordnen, in der faktischen Prioritätensetzung aber deutlich dahinter zurück bleiben. ${ }^{11}$ Denkbar ist, dass für die Zwecke der Abgeordneten weniger die tatsächlich durchgeführten Leistungen der Hilfe im Einzelfall als die Tatsache, dass sie regelmäßig angeboten - und angekündigt - werden, bedeutsam sind. So zeigte eine Bevölkerungsbefragung für SachsenAnhalt, dass Bürger - gefragt danach, wie sie ihren Landtagsabgeordneten erreichen können - am häufigsten auf die Bürgersprechstunden verweisen. ${ }^{12}$ Die im Rahmen von CITREP durchgeführte Bevölkerungsumfrage ergab, dass 16 Prozent der Befragten in Deutschland berichten, sie hätten in den vergangenen fünf Jahren Kontakt zu ihrem Bundestagsabgeordneten gehabt: Davon sagt aber nur gut ein Viertel (26 Prozent), dass dies im Rahmen von Bürgersprechstunden erfolgte (also vier Prozent der Befragten insgesamt), knapp zwei Drittel (62 Prozent) berichten von persönlichen Gesprächen bei Veranstaltungen. ${ }^{13}$

In der Selbstauskunft wird nur von einem Drittel der Abgeordneten als wichtige Tätigkeit im Wahlkreis das Erklären von Politik genannt (33 Prozent); fast schon unter ferner liefen rangiert nach eigener Angabe die Parteipflege (zehn Prozent). Die nähere Analyse der beobachteten Veranstaltungen zeigt, dass die Inhalte der Wahlkreisarbeit deutlich auch von Themen jenseits der Bundespolitik geprägt sind (vgl. Abbildung 2). Insbesondere die politische Situation und Sachthemen vor Ort nehmen viel Raum ein: Bei 49 Prozent der Veranstaltungen haben diese einen großen oder sehr großen Anteil, gefolgt von der Bundespolitik (35 Prozent), der Kommunal- (21 Prozent) und Landespolitik (20 Prozent). Abgeschlagen sind hingegen, wie schon gesagt, die Dienstleistungen für Einzelpersonen (zehn Prozent) sowie Themen der internationalen Politik (zwölf Prozent). Dies macht deutlich, dass Abgeordnete sich nicht auf die Bereiche ihrer formalen Zuständigkeit konzentrieren, sondern vielmehr mit allen Themen im föderal gestuften Mehrebenensystem der Bundesrepublik konfrontiert sind und diese bereitwillig „,bedienen“.

Die Bedeutung dieser „Mehrebenenrepräsentation“ zeigt sich nicht nur bei den behandelten Inhalten, sondern auch in der Zusammenarbeit mit Parlamentariern und Politikern anderer staatlicher Ebenen: Knapp die Hälfte der begleiteten MdBs (48 Prozent) traf im Rahmen ihrer Termine mit Landtagsabgeordneten zusammen. Es sind daher Zweifel angebracht an der Einschätzung, „hopes for close co-operation between members of the national parliament and of sub-national parliaments are generally groundless, because there is usually no reason for such co-operation" 14 .

Die Bundestagsabgeordneten entsprechen in der Schwerpunktsetzung und ihrem Verhalten in den Wahlkreisen grundsätzlich den Anreizen, die das personalisierte Verhältniswahlrecht setzt: Sie bieten vielfältige Kontaktmöglichkeiten im Einzelfall, beschränken sich nicht „bürokratisch" auf Themen ihrer formalen Zuständigkeit, sondern sind als bürgernahe Helfer auch bei der Unterstützung im Einzelfall und der Diskussion lokaler Themen sehr aktiv.

11 Vgl. Steffen H. Elsner / Karin Algasinger, „Sehr geehrte Frau Abgeordnete!“ - „Sehr geehrter Herr Abgeordneter!“. Der Bürger- und Wählerservice deutscher Abgeordneter zwischen Anspruch und Wirklichkeit. Ergebnisse einer experimentellen Fallstudie, München 2001.

12 Vgl. Bernhard Boll / Everhard Holtmann, Bürger und Parlament im Jahr 2000. Die Einstellungen der Landesbevölkerung zu Demokratie, Landtag und Landesregierung, Magdeburg 2000.

13 CITREP Bevölkerungsbefragung, 1.553 Befragte in Deutschland, 2010. Details zum Datensatz siehe die Beiträge von Oscar W. Gabriel und Mirjam Dageförde im vorliegenden Heft.

14 Werner J. Patzelt, The Constituency Roles of MPs at the Federal and Länder Levels in Germany, in: Regional and Federal Studies, 17. Jg. (2007), H. 1, S. 47 - 70, S. 67. 


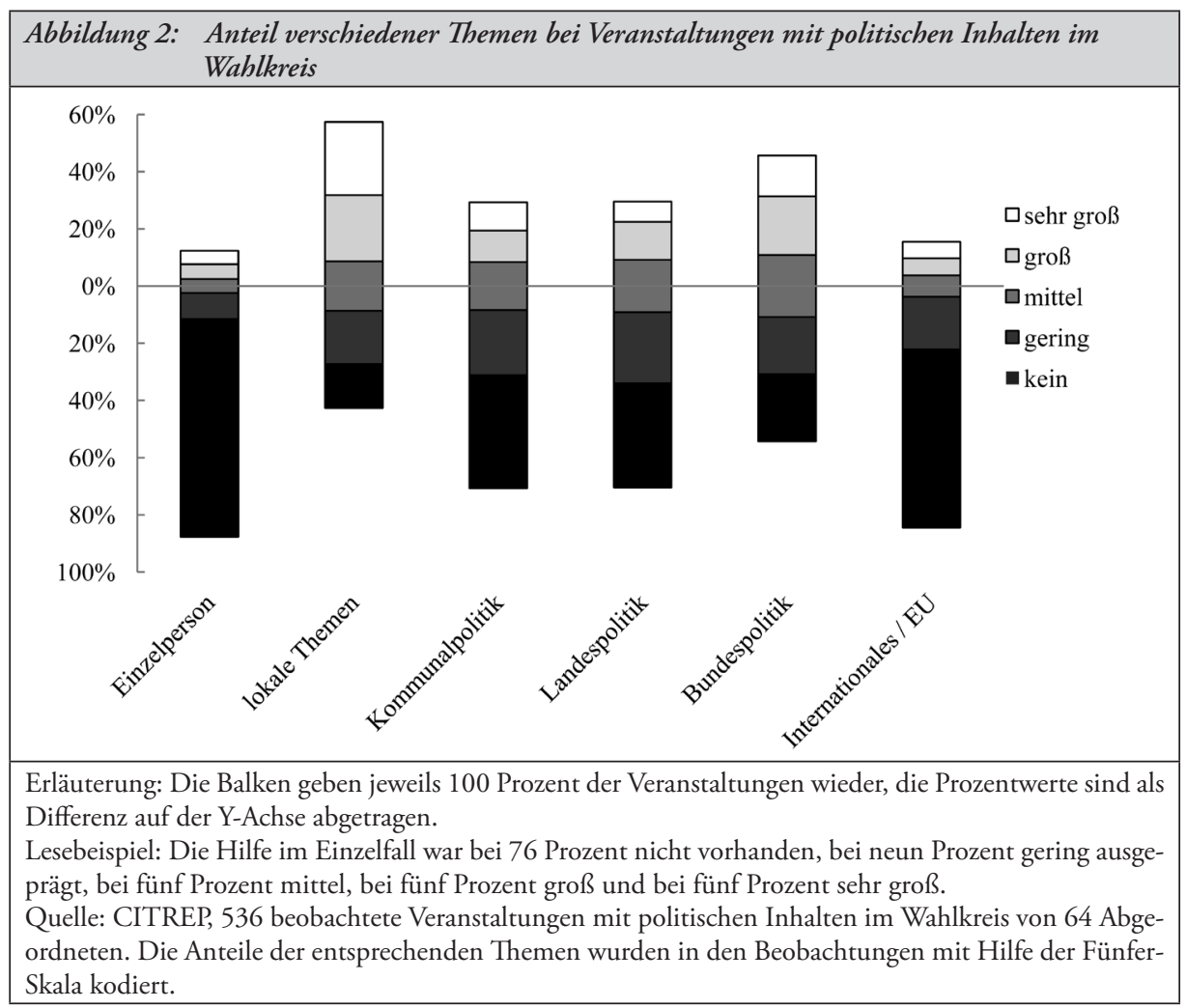

\subsection{Nominierung der Direktkandidaten durch die Parteiorganisationen im Wahlkreis}

Vor der Chance, gewählt zu werden, bedarf es der Aufstellung als Kandidat. Die Nominierung für die Direktkandidaten erfolgt in den Wahlkreisen durch die lokalen Parteiorganisationen, und zwar entweder durch eine Versammlung der Parteimitglieder oder von Delegierten. Das Delegiertenverfahren ist dabei weiterhin der übliche Weg; 70 Prozent aller Direktkandidaten sind 2009 so aufgestellt worden. ${ }^{15}$ Im Vorfeld der Nominierung ist es keine Seltenheit, dass die lokalen Parteiführungen versuchen, Einfluss auf das Kandidatenfeld zu nehmen. Den innerparteilichen Wahlakten gehen zudem oftmals sehr ausführliche interne Wahlkämpfe und Vorstellungen vor Ort im Rahmen der so genannten Tingeltouren voraus, in denen sich die Kandidaten bei den lokalen Parteigliederungen oder Parteimitgliedern im Wahlkreis persönlich vorstellen. Immer wieder kommt es vor - wenngleich dies natürlich Ausnahmeerscheinungen sind - dass etablierten Mitgliedern des Bundestages bei der Wiederaufstellung die Unterstützung vor Ort versagt wird. So unterlag im November 2012 der langjährige Abgeordnete Siegfried Kauder, CDU, mit nur 31 Prozent der Stimmen

15 Vgl. Marion Reiser, Wer entscheidet unter welchen Bedingungen über die Nominierung von Kandidaten? Die innerparteilichen Selektionsprozesse zur Aufstellung in den Wahlkreisen. Die Parteien nach der Bundestagswahl 2009, in: Oskar Niedermayer (Hrsg.), Die Parteien nach der Bundestagswahl 2009, Wiesbaden 2011, S. 237 - 259, S. 245. 


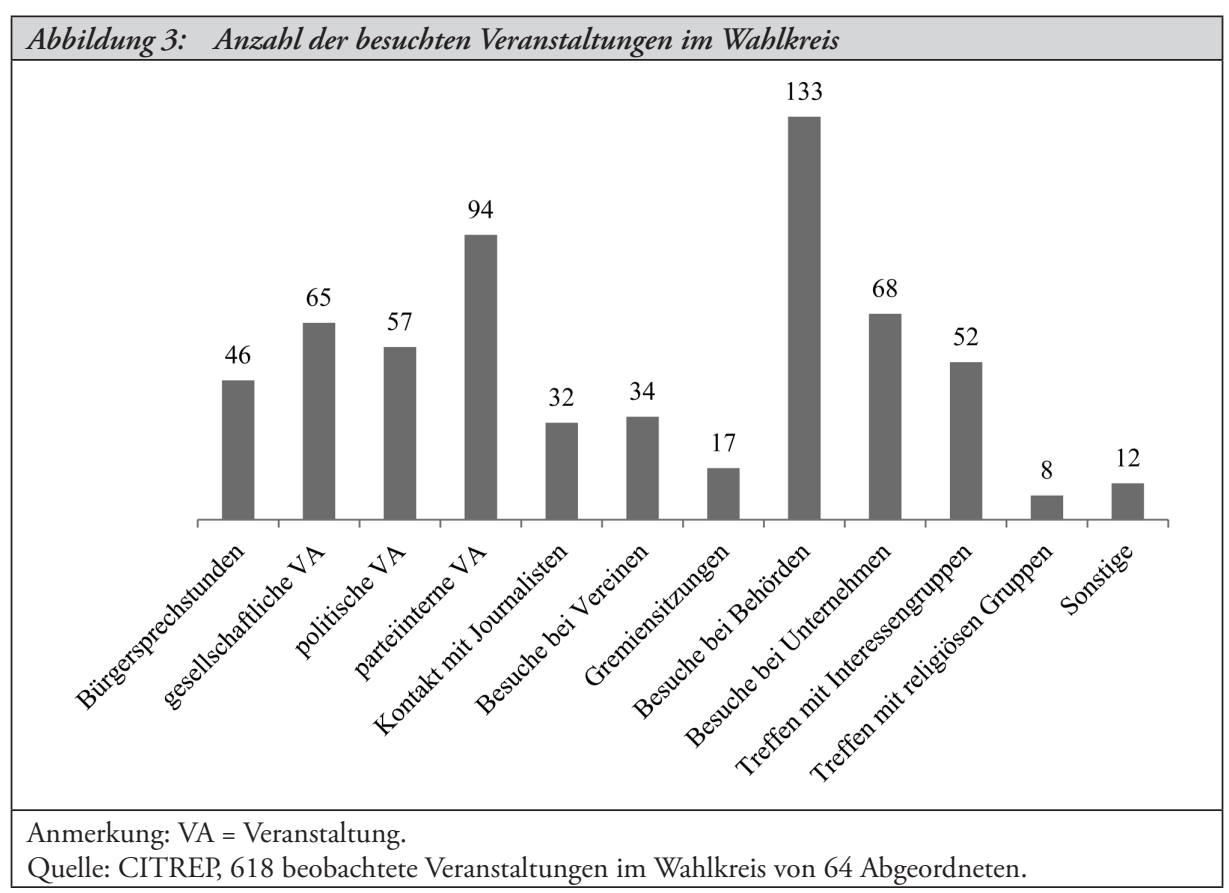

bei der Aufstellung seiner Partei für die Bundestagswahl 2013 dem jungen Oberbürgermeister von Donaueschingen, Torsten Frei (69 Prozent). Dies wurde auch auf den Stil der Wahlkreis- und Parteiarbeit Kauders zurückgeführt. ${ }^{16}$ Ohne Frage ist die lokale Parteiorganisation besonders wichtig für die Kür der Direktkandidaten. Dementsprechend ist davon auszugehen, dass direkt gewählte Abgeordnete in ihrer Wahlkreisarbeit großen Wert auf die Vernetzung mit der Partei vor Ort legen.

Der Blick auf die Veranstaltungen stützt diese Vermutung. Quantitativ steht deutlich an erster Stelle der Besuch von öffentlichen Einrichtungen wie Rathäusern, Schulen, Kindertagesstätten und ähnlichem (vgl. Abbildung 3). Betrachtet man die dort verbrachte Zeit näher, ziehen parteiinterne Veranstaltungen jedoch gleich mit den Behördenbesuchen (vgl. Abbildung 4). Die Parteiarbeit nimmt also in der Tat einen erheblichen Teil der im Wahlkreis verbrachten Zeit ein, der bei 19 Prozent der Gesamtbeobachtungsdauer lag. Diese große Bedeutung wird von den Abgeordneten in der Selbstauskunft weniger gewürdigt; nur sieben von 67 nennen sie überhaupt (vgl. Abbildung 1) - eine Zahl, die geringer ist als in früheren Befragungen: So berichtet Werner J. Patzelt, dass 82 Prozent der von ihm befragten Abgeordneten die Parteiarbeit auf regionaler und kommunaler Ebene als wichtig einstuften. ${ }^{17}$

Die große Wichtigkeit der Parteiarbeit ist im parlamentarischen Regierungssystem Deutschlands wenig überraschend, denn die Parteien nehmen die zentrale Funktion der Interessenvermittlung wahr. ${ }^{18}$ Ebenso wenig überraschend ist die Zurückhaltung bei der

16 Vgl. Rüdiger Soldt, Revolte im Schwarzwald, in: FAS vom 11. November 2012, S. 6.

17 Vgl. Werner J. Patzelt, Deutschlands Abgeordnete: Profil eines Berufsstands, der weit besser ist als sein Ruf, in: ZParl, 27. Jg. (1996), H. 3, S. 462 - 502, S. 484.

18 Vgl. den Beitrag von Danny Schindler im vorliegenden Heft. 


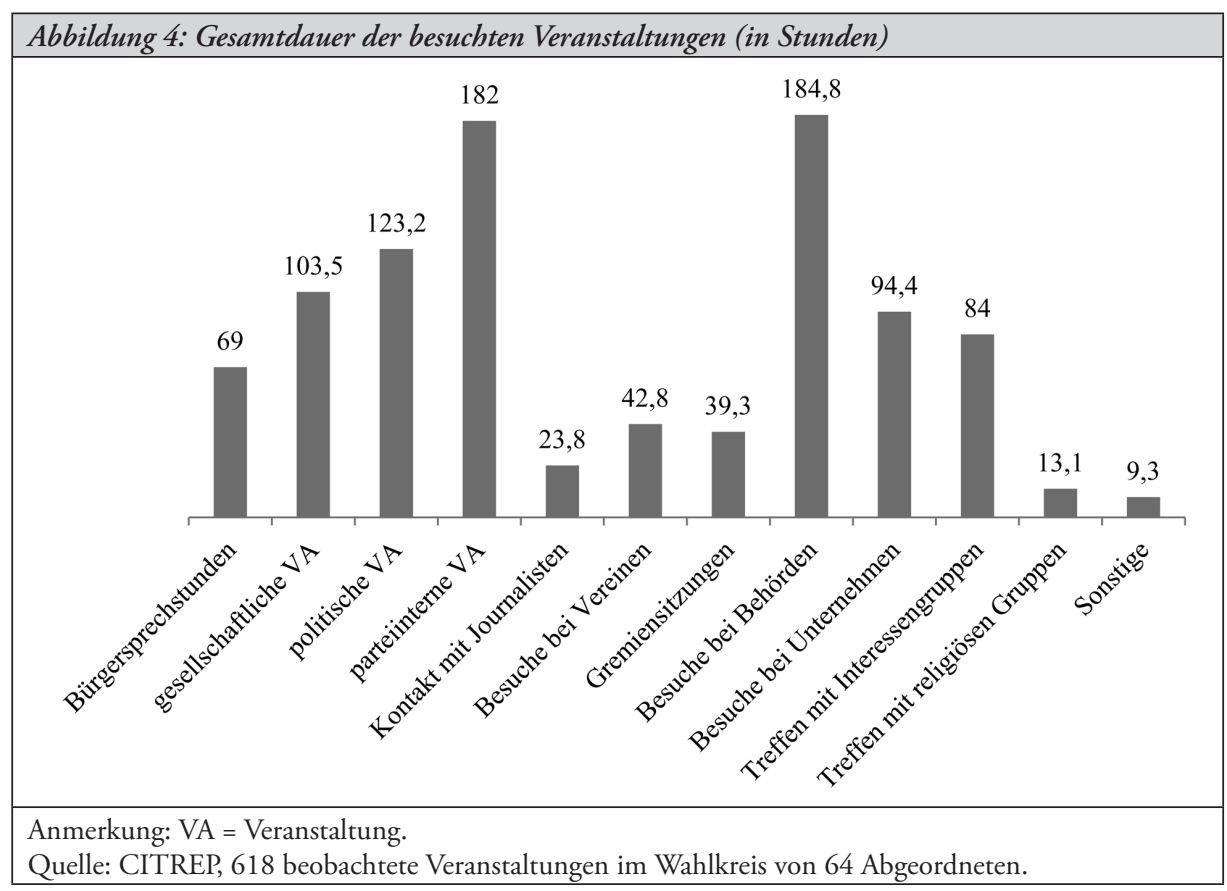

Außendarstellung dieser Aktivitäten, denn Anti-Parteien-Affekte und die Entfremdung von Parteiengagement sind in Deutschland schon lange etabliert. Dabei ist allerdings auch zu beachten, dass die Parteiarbeit im Wahlkreis keineswegs dominiert: Über 80 Prozent ihrer Zeit verbringen Abgeordnete dort nicht in geschlossenen Parteiveranstaltungen, sondern mit vielfältigen Aktivitäten und Besuchen bei Unternehmen, Vereinen etc. vor Ort.

In der internationalen Literatur ist teilweise die Einschätzung vertreten worden, dass aufgrund des Nominierungsverfahrens die Anreizstrukturen für über Parteilisten gewählte Abgeordnete grundsätzlich anders seien und somit auch zu einer anderen Schwerpunktsetzung in der Parlaments- und Wahlkreisarbeit führten: Weil die Landeslisten auf Landesdelegiertenkonferenzen aufgestellt werden, sei die Bedeutung der Vernetzung in der Landespartei noch größer, da für den Einzug ins Parlament nur ein aussichtsreicher Listenplatz benötigt werde, nicht aber der tatsächliche Sieg in der Wahl vor Ort; Listenabgeordnete "depend on their state party, not on voters, for a seat in the legislature "19. Entsprechend zeigt eine quantitative Analyse für die Jahre 1990 bis 2002, dass in Ausschüssen des Bundestages, mit deren Zuständigkeitsbereich stärker Wahlkreisinteressen bedient werden können (zum Beispiel die Ausschüsse für Verkehr und Landwirtschaft), mehr Mitglieder mit Direktmandat sitzen als in solchen, bei denen dies kaum möglich sei (zum Beispiel wirtschaftliche Zusammenarbeit und Verteidigung). Es gebe daher signifikante Unterschiede zwischen Direkt- und Listenabgeordneten. ${ }^{20}$ Allerdings wurde auch gezeigt, dass Ausschussmitglied-

19 Thomas Stratmann / Martin Baur, Plurality Rule, Proportional Representation, and the German Bundestag: How Incentives to Pork-Barrel Differ across Electoral Systems, in: American Journal of Political Science, 46. Jg. (2002), H. 3, S. 506 - 514, S. 508.

20 Vgl. ebenda, S. 513. 
schaften starken Schwankungen unterliegen und der Zeitraum von 1990 bis 2002 für Verallgemeinerungen unzureichend ist. ${ }^{21}$

Auf Basis von Selbstauskünften wurde überdies festgestellt, dass 99 Prozent der Wahlkreisabgeordneten, aber nur 84 Prozent der Listenabgeordneten angeben, wöchentlichen Kontakt zu Bürgern in ihrem Wahlkreis zu haben. ${ }^{22}$ Eine gründliche Analyse von Interviewdaten mit Abgeordneten aus Bundestag und Landtagen macht in der Tat auch in der Rollenorientierung Unterschiede sichtbar: So haben direkt gewählte Abgeordnete insgesamt etwas stärker die Wahlkreisarbeit betont, dabei nach eigener Auskunft mehr gesellschaftliche Veranstaltungen mit hoher Sichtbarkeit besucht, mehr Bürgerservice geleistet und sich stärker um lokale Projekte gekümmert. ${ }^{23}$ Doch handelt es sich erstens um nur geringe Unterschiede, und zweitens lassen diese sich nicht systematisch von anderen Faktoren - insbesondere der Größe der Partei - trennen. ${ }^{24}$

Betrachtet man die Nominierungsprozesse für die Landeslisten näher, so stellt sich zudem heraus, dass es faktisch mittlerweile für eine aussichtsreiche Positionierung auf der Landesliste Voraussetzung ist, auch direkt anzutreten. In der 15. Wahlperiode waren 94 Prozent der Abgeordneten, die über die Parteilisten in den Bundestag eingezogen sind, auch als Direktkandidaten im Rennen gewesen. ${ }^{25}$ Die Aufstellung in einem Wahlkreis ist daher für so gut wie alle Abgeordneten erforderlich. Gestützt wird dies durch Befunde zu Karriereverläufen von Bundestagsabgeordneten zwischen 1949 und 2009: Nur 23 Prozent aller MdBs können ausschließlich entweder als Listen- oder Wahlkreisabgeordnete klassifiziert werden; die anderen sind im Laufe ihrer Mandatszeiten das eine oder andere oder eben beides zugleich gewesen. ${ }^{26}$ Es ist also davon auszugehen, dass sich die direkt und über Liste gewählten Abgeordneten vielleicht in Details, nicht aber grundsätzlich unterscheiden und beide ihre Wahlkreisarbeit in ähnlicher Form betreiben. ${ }^{27}$

Von den 67 interviewten MdBs hatten 52 Prozent ein Direktmandat (genau wie im 17. Bundestag insgesamt) und 48 Prozent waren über eine Parteiliste eingezogen. Dennoch hatten bis auf einen „Nachrücker“ alle Abgeordneten einen eigenen Wahlkreis (also 98 Prozent), in dem sie angetreten waren und den sie als den ihren verstanden und vertraten. Eine erste Auswertung zeigt, dass sich das Repräsentationsverhalten der Abgeordneten mit den beiden

21 Vgl. Dominic Heinz, Mandatstypen und Ausschussmitgliedschaften der Mitglieder des Deutschen Bundestages - Eine empirische Untersuchung von 1949 bis 2005, in: ZParl, 41. Jg. (2010), H. 3, S. $518-527$, S. 526.

22 Vgl. Hans-Dieter Klingemann / Wolfgang Wessels, The Consequences of Germany's Mixed Member System: Personalization at the Grassroots?, in: Matthew Soberg Shugart / Martin P. Wattenberg (Hrsg.), Mixed-Member Electoral Systems. The Best of Both Worlds?, Oxford 2001, S. 279 - 296, S. 292.

23 Vgl. Werner J. Patzelt, The Constituency Roles of MPs at the Federal and Länder Levels in Germany, in: Regional and Federal Studies, 17. Jg. (2007), H. 1, S. $47-70$.

24 Vgl. ebenda, S. 58.

25 Vgl. Suzanne S. Schüttemeyer / Roland Sturm, Der Kandidat - das (fast) unbekannte Wesen: Befunde und Überlegungen zur Aufstellung der Bewerber zum Deutschen Bundestag, in: ZParl, 36. Jg. (2005), H. 3, S. $539-553$, S. 548 f.

26 Vgl. Philip Manow, Wahlkreis- oder Listenabgeordneter, Typus oder Episode? Eine Sequenzanalyse der Wege in den Bundestag, in: PVS, 53. Jg. (2012), H. 1, S. 53 - 78, S. 75.

$27 \mathrm{Ob}$ es sich dabei um einseitige „Kontaminationseffekte“ der Mehrheitswahl auf die Listenwahl im Wahlrecht Deutschlands handelt, ist für die politische Praxis wenig bedeutsam, vgl. Federico Ferrara / Erik S. Herron / Misa Nishikawa, Mixed Electoral Systems: Contamination and its Consequences, New York 2005. 


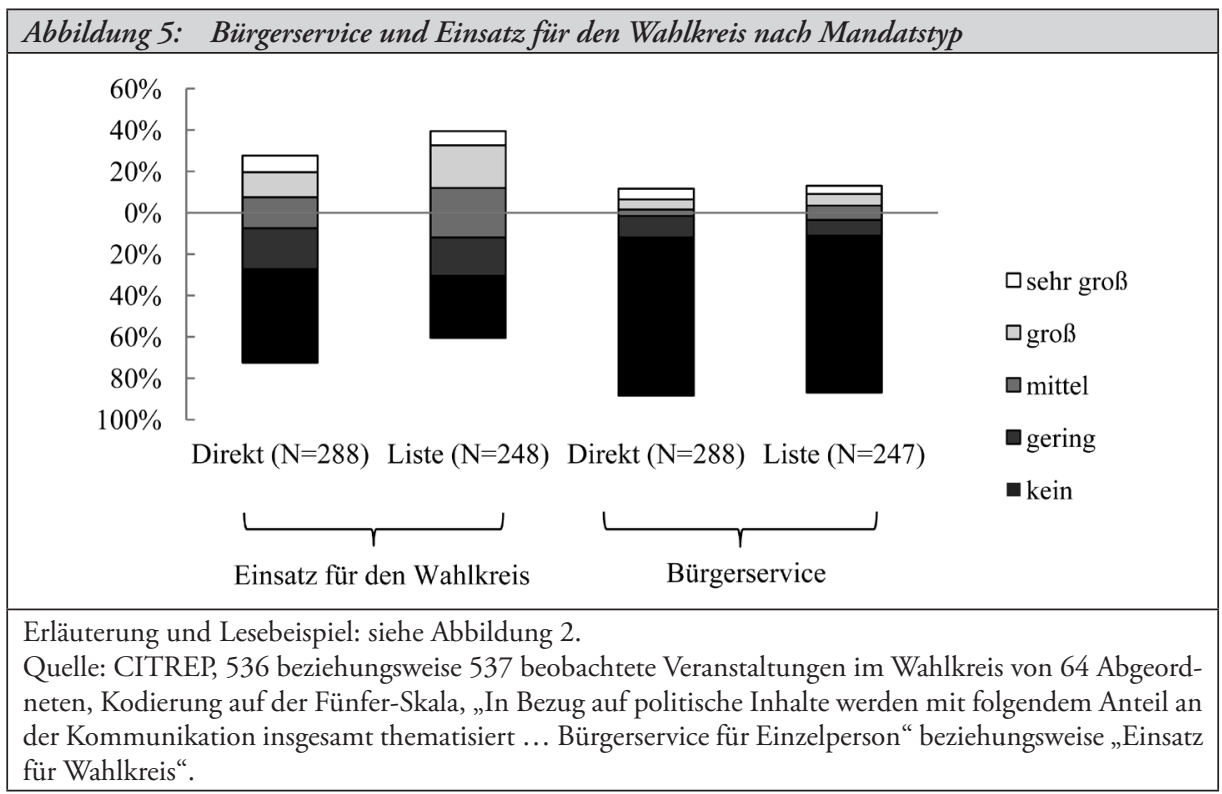

Mandatstypen nur marginal unterscheidet und zwar teilweise entgegen der in der Literatur geäußerten Annahme einer größeren Bürgernähe von direkt gewählten Abgeordneten beziehungsweise einer größeren Parteinähe von über Liste gewählten (vgl. Abbildung 6):

- Die direkt gewählten Abgeordneten besuchten in ihren Wahlkreisen einen etwas höheren Anteil an gesellschaftlichen Veranstaltungen (zwölf Prozent) als die über Liste eingezogenen (acht Prozent). Doch der Anteil der Parteiveranstaltungen ist bei beiden identisch (jeweils 15 Prozent).

- Während der Veranstaltungen zeigten über die Liste gewählte Abgeordnete einen höheren Einsatz für ihren Wahlkreis: 27 Prozent verwendeten einen (sehr) großen Anteil der Kommunikation darauf, während dies nur 20 Prozent der direkt gewählten Abgeordneten taten (vgl. Abbildung 5).

- Beim Bürgerservice hingegen gab es keinen nennenswerten Unterschied (großer und sehr großer Anteil bei jeweils zehn Prozent der Veranstaltungen).

- Der Anteil von Bürgersprechstunden ist für Abgeordnete mit beiden Mandatsarten etwa gleich (jeweils sieben Prozent).

Insgesamt ist als Ergebnis dieser ersten Übersicht festzuhalten, dass es zwar Unterschiede zwischen Abgeordneten mit Listen- und Direktmandat gibt, diese sich jedoch in minimalen Größenordnungen bewegen und teilweise auch den gängigen Annahmen in der Literatur widersprechen.

Es bedarf genauerer auch multivariater Analysen, doch der erste Eindruck bestärkt die Vermutung, dass es sich bei der These von zwei grundsätzlich unterschiedlichen Typen von Abgeordneten mit differierendem Repräsentationsverhalten um ein weiteres „Ammenmärchen" der Parlamentsforschung handeln dürfte. ${ }^{28}$

28 Vgl. Stephen Padgett / Tony Burkett, Political Parties and Elections in West Germany. The Search for a New Stability, London 1986, S. 130. 


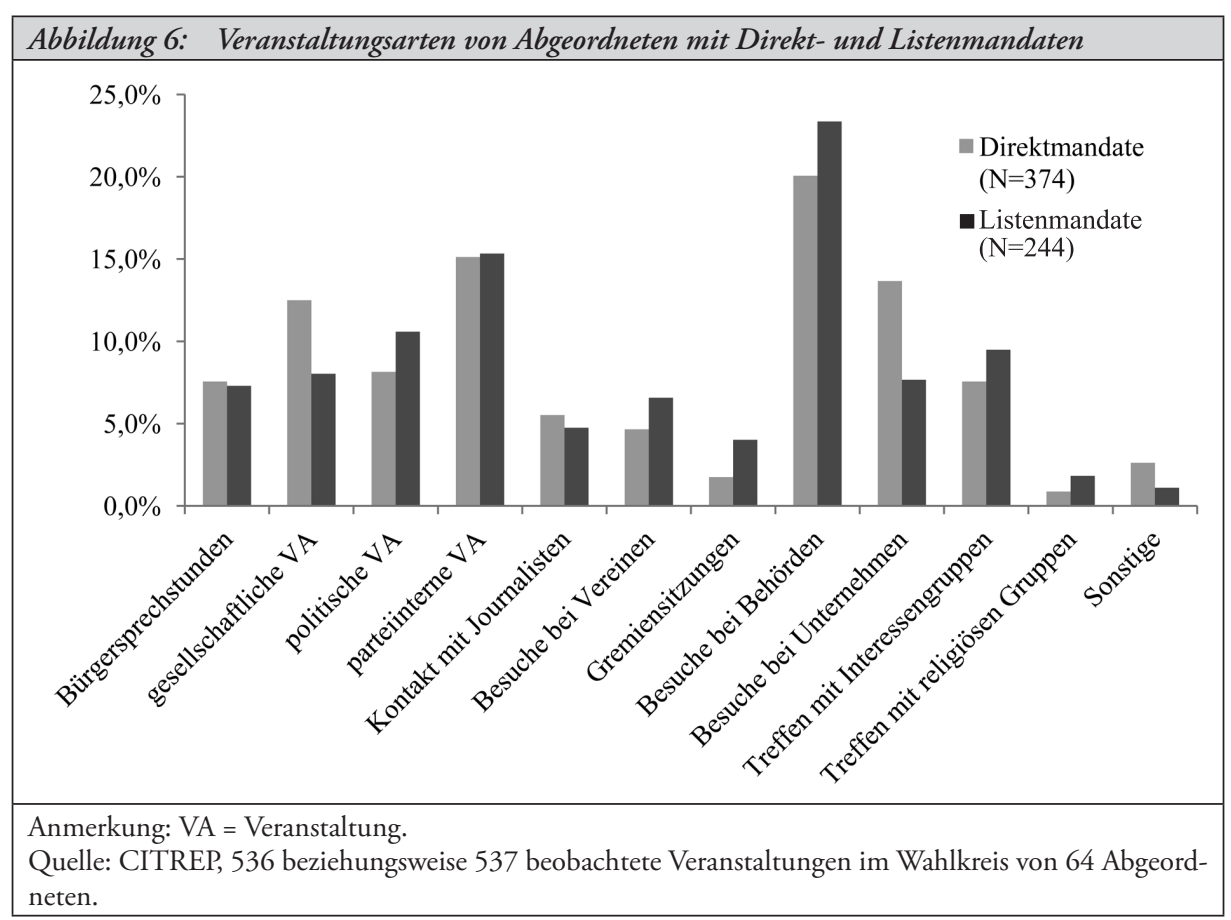

In Bezug auf die Anreize aus dem System der Kandidatenaufstellung zeigt sich, dass sich die Abgeordneten klar daran ausrichten: Die Pflege der Parteibasis vor Ort spielt eine wesentliche Rolle bei der Wahlkreisarbeit, ohne dass die Abgeordneten sich allein auf die Parteiarbeit beschränken. Der These einer Rollendifferenzierung von Abgeordneten mit Direkt- und Listenmandat muss indes wiederum widersprochen werden, was allerdings bei näherer Betrachtung der entsprechenden faktischen - nicht nur formalen - Anreize kaum überraschend ist.

\subsection{Informationsquelle für die Parlamentsarbeit}

Als Arbeitsparlament hat der Bundestag eine hochgradig ausdifferenzierte Arbeitsteilung zwischen thematisch spezialisierten Abgeordneten entwickelt. Die Ressortstruktur der Bundesministerien ist durch die Aufteilung der Fachausschüsse gespiegelt, die ihrerseits in den Fraktionen durch Arbeitskreise abgebildet wird. ${ }^{29}$ Der Bundestag ist somit zutreffend als Fraktionenparlament wie auch als Expertenparlament bezeichnet worden, in dem jeder Abgeordnete Spezialist für ein - oder mehrere - Politikfelder ist. ${ }^{30}$ In dieser Tätigkeit nutzt er vielfältige Informationsquellen von Fachgesprächen mit Ministerien und Interessengruppen über Ausschussanhörungen bis zu Informationsreisen. Vor dem Hintergrund dieser für den

29 Vgl. Suzanne S. Schüttemeyer, Fraktionen im Deutschen Bundestag 1949-1997. Empirische Befunde und theoretische Folgerungen, Opladen 1998, S. $85 \mathrm{ff}$.

$30 \mathrm{Vgl}$. Jürgen von Oertzen, Das Expertenparlament. Abgeordnetenrollen in den Fachstrukturen bundesdeutscher Parlamente, Baden-Baden 2006. 
Parlamentsbetrieb konstitutiven Spezialisierung kann vermutet werden, dass die Wahlkreisarbeit als eine ergänzende „Pflicht“ wahrgenommen wird, die aber eher aus elektoralem Kalkül denn aus substantiellen Motiven übernommen wird. Insofern wäre zu erwarten, dass die Abgeordneten die Parlaments- und die Wahlkreisarbeit als zwei unterschiedliche Seiten derselben Medaille verstehen.

Um sich der Verbindung dieser beiden Seiten im Selbstverständnis der MdBs zu nähern, wurden sie nach deren Bedeutung füreinander gefragt. Dabei bezeichneten zwei Drittel (43 von 65, entspricht 66 Prozent) die Wahlkreisarbeit als sehr wichtig für die Parlamentstätigkeit, umgekehrt wurde dies nur von 29 (47 Prozent) so gesehen; lediglich neun MdBs (14 Prozent) maßen ihrer Arbeit vor Ort eine geringe Bedeutung für jene im Parlament bei, aber 19 (31 Prozent) hielten ihre fachlichen Schwerpunktsetzungen im Bundestag für eher unwichtig im Wahlkreis.

Die vertiefte Auswertung dieser Antworten kann mit Hilfe des systemtheoretischen Modells von David Easton unterteilt werden in Input (Support und Demands) und Feedback ${ }^{31}$ : Fast die Hälfte der Abgeordneten betonen, dass die Wahlkreisarbeit ihnen wichtige Rückmeldung gebe, insbesondere zu laufenden Debatten und Gesetzesvorhaben, aber auch zu bereits geltenden Gesetzen. Die folgenden Zitate illustrieren diese Rückmeldungsfunktion:

„Ich denke, dass Wichtigste ist einfach, dass man vor Ort die Auswirkungen von politischen Entscheidungen mitbekommt beziehungsweise wie Debatten in Berlin vor Ort ankommen, um dies gut reflektieren zu können und dann mit diesen Erfahrungen nach Berlin zurückzukommen und dort diskutieren zu können. Das ist dann der Praxistest, den man in sitzungsfreien Wochen vollziehen kann." (Stephan Kühn, MdB, Bündnis 90/Die Grünen)

„Die Wahlkreisarbeit finde ich deshalb wichtig, weil sie praktische Erkenntnisse liefert. Gesetzesarbeit ist Arbeit an einem Rahmen, und der Rahmen kann passen, oder er kann zu eng oder zu groß sein. Das tägliche Anwenden von Regelungen, die der Bundesgesetzgeber geschaffen hat, zeigt, wie gut oder wie schlecht Gesetze sind, zeigt Handlungsbedarf auf. Wer diese praktischen Erfahrungen geschildert bekommt, nur der wird merken und erkennen können, wo er handeln, wo er nachjustieren muss." (Annette Widmann-Mauz, MdB, CDU)

Etwa ein Viertel der Gesprächspartner betont, im Wahlkreis könne gut die Stimmung der Menschen aufgenommen werden. Wiederum knapp die Hälfte stellt die Input-Funktion in den Vordergrund, in Form von konkreten Forderungen oder allgemeinen Informationen. Erneut zeigt sich (vgl. Abbildung 1), dass für die Abgeordneten bei der Wahlkreisarbeit deutlich im Vordergrund steht, Informationen, Stimmungen und Reaktionen vor Ort zu sammeln und aufzunehmen (vgl. Abbildung 7).

Auch die Beobachtungsdaten stützen dies. So sind, wie bereits berichtet, der Großteil der Veranstaltungen Besuche bei Behörden und Verwaltungseinrichtungen im Wahlkreis, die nicht geeignet sind, eine große Breitenwirkung zu erzielen, zumal die Zahl der Gesprächsteilnehmer gering ist: Bei der Hälfte dieser Veranstaltungen hat der Abgeordnete weniger als sechs Gesprächspartner, bei 78 Prozent weniger als zehn. ${ }^{32}$ Dabei handelt es sich meist um die Hausleitung und teilweise auch Personalräte oder Fachleute zu bestimmten Themenbereichen. Die Auswertung der Unternehmensbesuche ergibt ein ähnliches Bild: Zwei Drittel der Veranstaltungen haben bis zu fünf Teilnehmer, 79 Prozent bis zu zehn. Es ist also kaum die unmittelbare Kontaktaufnahme zu potenziellen Wählern, die hier im Vordergrund steht, sondern

31 Vgl. David Easton, A Systems Analysis of Political Life, New York 1965.

32 Quelle: CITREP, basierend auf 567 entsprechend kodierten Veranstaltungen. 


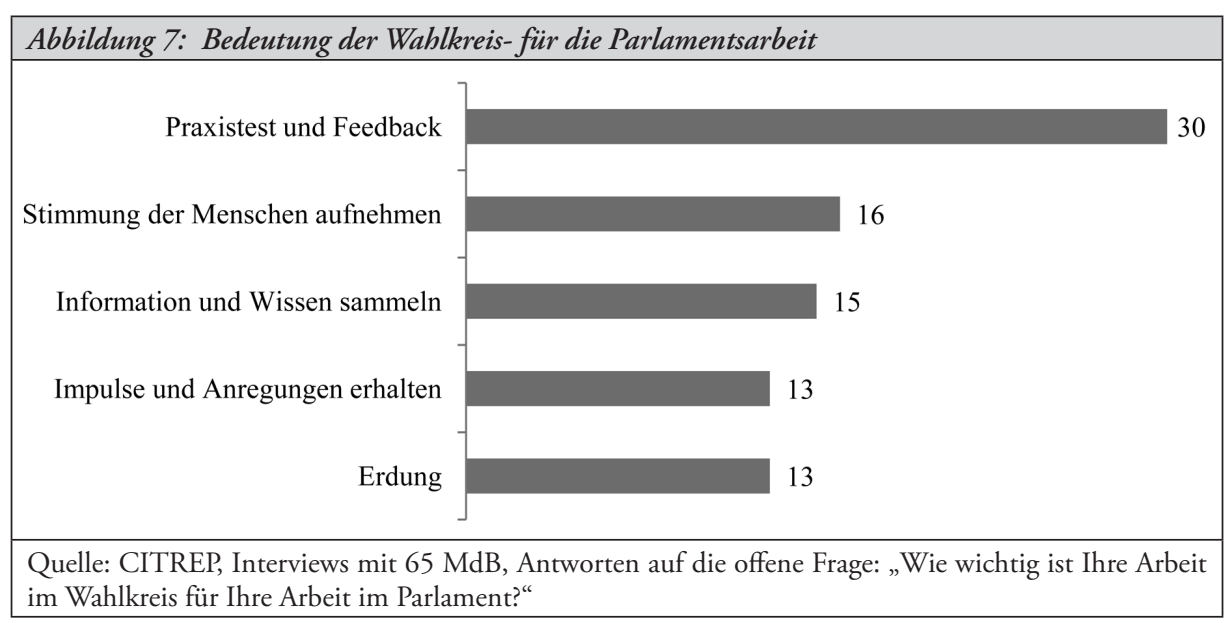

viel wichtiger dürften eben die Informationsaufnahme und die Vernetzung vor Ort sein.

Die Auswertung der Veranstaltungsdauer deutet in dieselbe Richtung: Die Termine dauern durchschnittlich 1,6 Stunden, lassen also durchaus einen substantiellen Austausch zu. Nur bei einem Zehntel der Fälle bleiben die Abgeordneten weniger als eine halbe Stunde (vgl. Abbildung 8). Nach einem vollen Tag mit zwölf Wahlkreisterminen schaute eine Abgeordnete entsprechend kurz für 20 Minuten bei einem Vereinsfest vorbei, musste dann aber weiter zum schlecht verschiebbaren Termin des Fastenbrechens der muslimischen Gemeinde. Dem Beobachter erläuterte sie entschuldigend, normalerweise bleibe sie nicht so kurz, denn ,wenn man sich die Zeit nicht nimmt, wirkt der Auftritt so, als würde man grad mal vorbeischauen ... so typisch Politiker" (MdB, anonym).

Natürlich variiert die Dauer der verschiedenen Veranstaltungsarten erheblich: Besonders lang sind Gremiensitzungen (durchschnittlich 2,3 Stunden), politische Veranstaltungen (2,1 Stunden) und Parteiveranstaltungen (1,9 Stunden), kürzer fallen Journalistenkontakte aus (42 Minuten). Dies liegt aber zum Teil in der Natur der Sache begründet und kann kaum als verschiedene Prioritätensetzung interpretiert werden: Ein Journalistengespräch ist üblicherweise eine Vier-Augen-Veranstaltung mit hohen Redeanteilen des Abgeordneten, während Gremiensitzungen in einem engeren vorgegebenen Korsett mit vielen weiteren Teilnehmern verlaufen.

Dass es bei der Wahlkreisarbeit den Abgeordneten vor allem darum geht, sich Informationen zu beschaffen - wie sie selbst sagen - , wird auch durch das Verhalten während der beobachteten Veranstaltungen gestützt: Stark und sehr stark ausgeprägt war die Informationsaufnahme bei 60 Prozent der begleiteten Veranstaltungen, die Vermittlung der eigenen Position hingegen nur bei 36 Prozent (vgl. Abbildung 9).

Die insgesamt große Bedeutung der Informationsaufnahme bei der Wahlkreisarbeit in der Selbsteinschätzung wie im realen Verhalten verweist auch auf Prozesse der innerparlamentarischen Arbeitsteilung. Denn offensichtlich ist auch, dass es hier nicht ausschließlich um Input und Feedback zur eigenen parlamentarischen Spezialisierung gehen kann. Vielmehr werden vielfältige aktuell relevante Themenbereiche angesprochen, wie folgende Zitate verdeutlichen: 


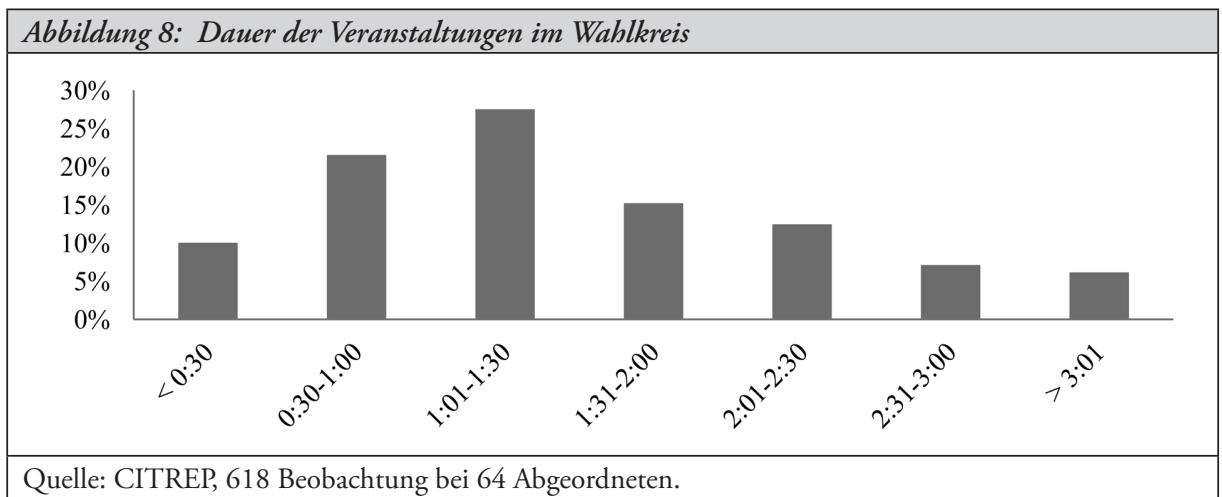

„Ich habe Glück, dass ich mit dem Fachausschuss Gesundheit ein Themengebiet anspreche, was sehr viele Leute interessiert. Aber ansonsten muss ich im Wahlkreis eben auch Generalist sein, muss auskunftsfähig sein über die brennenden Themen, die in den überregionalen Medien oder in der Tagesschau eine Rolle spielen." (Jens Ackermann, MdB, FDP)

„Der Parlamentarier ist Generalist. Er entscheidet hier im Parlament über alles - vom Bundeswehreinsatz bis zum Euro-Rettungsschirm ist er für alles zuständig. In seinem Ausschuss ist er aber nur in einem Fachgebiet tätig. Das heißt, dass er draußen meistens lediglich als Fachpolitiker wahrgenommen wird. In seinem Wahlkreis muss er jedoch die Fragen zu allen Themen beantworten und auch hier im Bundestag zu allen Themen entscheiden. Das heißt, der Abgeordnete ist Generalist und muss im Parlament gleichzeitig Spezialist sein, weil er hier auch sonst gar nicht mitreden kann." (Eduard Oswald, MdB, CSU)

\section{Abbildung 9: Anteile der Darstellung eigener Positionen und Aufnahme von Informationen}

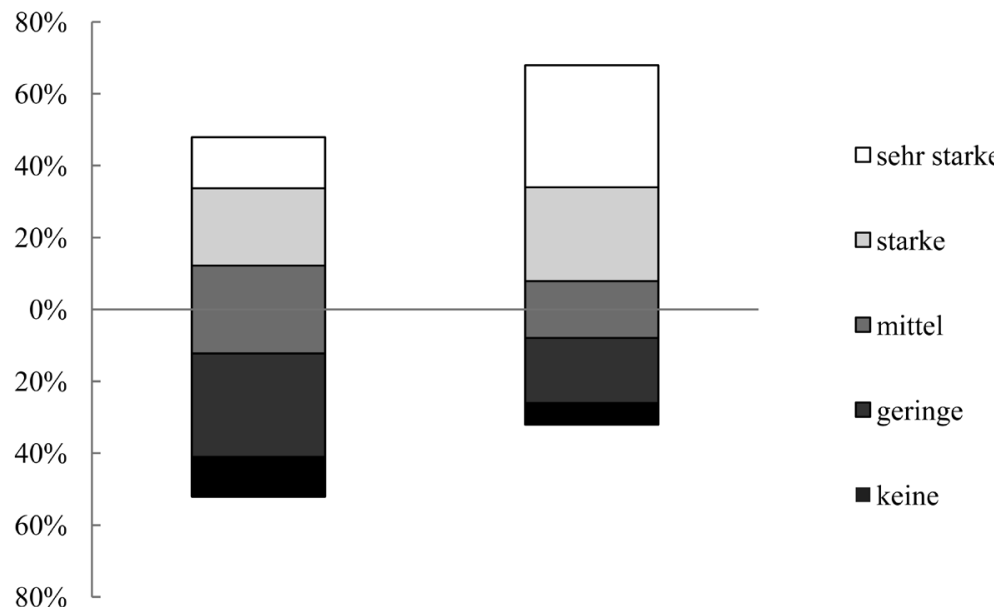
Darstellung / Vermittlung eigener Position
Aufnahme von
Informationen

Erläuterung und Lesebeispiel: siehe Abbildung 2.

Quelle: CITREP, Beobachtung von 479 beziehungsweise 480 Veranstaltungen und Kodierung der jeweiligen Anteile auf der Fünfer-Skala. 
Diese Unterteilung von Generalist im Wahlkreis und Spezialist in Berlin, die sich auch in politikwissenschaftlichen Lehrbüchern findet ${ }^{33}$, wird wortgleich von zahlreichen Abgeordneten aufgenommen. Die weitere Bearbeitung der aufgenommenen Informationen liegt mithin in der Organisation der Fraktionsarbeit. Dabei kann von einem mehrstufigen Prozess der Berücksichtigung und Filterung ausgegangen werden, der von den Bürgern im Wahlkreis zu ihren jeweiligen Abgeordneten, weiter (über direkte Kontakte und Aussprachen in der Fraktionsversammlung) zu den Fachexperten der eigenen Fraktion, damit in die Arbeitskreise der Fraktionen und von dort in die Ausschüsse verläuft. Dieser Prozess dient somit auch der Kontrolle der eigenen Abgeordneten-Kollegen, die in der Fraktion ein anderes Fachgebiet bearbeiten ${ }^{34}$ :

„Die Ergebnisse aus den Gesprächen, die ich im Wahlkreis bei Veranstaltungen oder bei anderen Gesprächsrunden führe, nehme ich selbstverständlich mit nach Berlin. Ich kommuniziere sie, soweit es nicht mein eigenes Sachgebiet betrifft, an die entsprechenden Kollegen weiter." (Angelika Graf, MdB, SPD)

„Die Erfahrungen, die ich im Wahlkreis sammle, und auch die Themen, die an mich im Wahlkreis herangetragen werden, fließen in die Gespräche ein, die man im Bundestag (vor allem in der Fraktion) mit Kollegen führt." (Stephan Thomae, MdB, FDP)

Es wird zu vertiefen sein, inwieweit die Unterteilung von Generalist und Spezialist geeignet ist, um als Grundlage für eine Rollentypologie der Abgeordneten zu dienen. ${ }^{35}$ Insbesondere ist zu prüfen, ob das Konzept von „Hill-Style“ und „Home-Style“ nach Richard S. Fenno für die deutschen Abgeordneten fruchtbar ist ${ }^{36}$, wie es in der einen oder anderen Form schon von Uwe Thaysen („Bonn-Stil vs. Basis-Stil“) und Werner J. Patzelt („Parlamentsstil vs. Wahlkreisstil“) angedeutet wurde. ${ }^{37}$

Dass die Abgeordneten ihre Wahlkreisarbeit umfänglich für die Aufnahme von Stimmungen, Rückmeldungen und Informationen zu verschiedensten politischen Themen nutzen, mag im System der parlamentarischen Arbeitsteilung zunächst überraschen, da es den Anreizen der Spezialisierung nicht direkt entspricht. Verständlich wird dieses Verhalten jedoch in Verbindung mit den Anreizen zur Wahl - schließlich wird es von einem Abgeordneten erwartet, dass er zu allen Themen auskunftsfähig ist - sowie als ein innerfraktionelles Instrument zur Kontrolle der eigenen Fachexperten.

33 Vgl. Wolfgang Ismayr, Der Deutsche Bundestag im politischen System der Bundesrepublik Deutschland, Opladen 2001, S. 45.

34 Vgl. Danny Schindler / Sven T. Siefken, Wahlkreisarbeit der Abgeordneten des Deutschen Bundestages - ein Beitrag zur Parlamentarischen Kontrolle?, in: Birgit Eberbach-Born / Sabine Kropp I Andrej Stuchlik / Wolfgang Zeh (Hrsg.), Parlamentarische Kontrolle und Europäische Union, Baden-Baden 2013, S. 203 - 225.

35 Vgl. Sven T. Siefken, German MPs' Styles of Representation in the District, Vortrag im Rahmen der DVParl-Konferenz „Repräsentation im Wahlkreis - Bevölkerung und Abgeordnete in Deutschland und Frankreich“, Berlin am 7./8. Dezember 2012.

36 Vgl. Richard F. Fenno, Home Style, a.a.O. (Fn. 1).

37 Vgl. Uwe Thaysen, Representation in the Federal Republic of Germany, in: ders. I Roger H. Davidson / Robert Gerald Livingston (Hrsg.), The U.S. Congress and the German Bundestag. Comparisons of Democratic Processes, Boulder 1990, S. 67 - 107, S. 90; Werner J. Patzelt, Abgeordnete und Repräsentation: Amtsverständnis und Wahlkreisarbeit, Passau 1993, S. 78. 


\subsection{Kommunikationsforum in der Mediendemokratie}

Eine weitere Funktion der Wahlkreisarbeit ist es, ein Forum für direkte Kommunikation von Repräsentanten und Repräsentierten zu bieten. In modernen Großdemokratien ist eine umfassende persönliche Interaktion mit den Repräsentierten nur schwer möglich. Jeder Wahlkreis in Deutschland hat etwa 250.000 Einwohner; schon rein rechnerisch ist direkter Kontakt mit ihnen allen ausgeschlossen: Wollte ein Abgeordneter auch nur zehn Minuten mit jedem Bürger sprechen und dafür acht Stunden pro Tag zur Verfügung stellen, wäre dies in einer Wahlperiode gar nicht zu bewerkstelligen: Er bräuchte dafür über sieben Jahre ohne Urlaub, ohne Feiertage, ohne Krankheit - und ohne Parlamentsarbeit. Zumindest können Abgeordnete aber versuchen, ein möglichst breites Angebot zur Kommunikation zu schaffen über die Teilnahme an gesellschaftlichen Veranstaltungen oder die Ansprache von Multiplikatoren. Insbesondere sind hierfür die Massenmedien vor Ort sowie die neuen Medien wichtig.

Angesichts der generellen Einschätzung, dass es zu einer Zunahme der Bedeutung von Massenmedien im politischen Prozess komme ${ }^{38}$, ist mit einer entsprechenden Schwerpunktsetzung der Parlamente allgemein und der Abgeordneten bei ihrer Wahlkreisarbeit im Speziellen zu rechnen. Beides ist indes bislang kaum erforscht. ${ }^{39}$ Basierend auf Tiefeninterviews mit Abgeordneten zu ihrem Kommunikationsverhalten hat Annette Knaut gezeigt, dass keineswegs der neue Typ des „Medienpolitikers“ vorherrsche, sondern weiterhin vor allem der traditionelle Kommunikationsstil gepflegt wird, der persönliche Kommunikation und die Vernetzung vor Ort in den Vordergrund stellt, oder aber ein Stil, der situationsgebunden wechselt, ohne dass eine klare Präferenz feststeht („Netzwerkstil“). ${ }^{40}$

Nach der Häufigkeit unterschiedlicher Kommunikationskanäle befragt, zeigt sich auch in der vorliegenden Untersuchung, dass die Abgeordneten ihre Wahlkreisarbeit nicht sehr stark durch Medienarbeit geprägt sehen: Zwar gab etwa die Hälfte (52 Prozent) an, dass sie zur Kommunikation regelmäßig Printmedien nutze (nur neun Prozent berichten vom Rundfunk). Doch bei dem beobachteten Verhalten waren nur fünf Prozent der Veranstaltungen Journalisten-Gespräche (vgl. Abbildung 3), und nur bei einem guten Viertel der anderen Termine waren Journalisten anwesend (27 Prozent). ${ }^{41}$ Demgegenüber betonten 89 Prozent der Abgeordneten die besondere Bedeutung von persönlicher Kommunikation bei Besuchen vor Ort, 82 Prozent die Abstimmung via Brief, E-Mail oder Telefon und 80 Prozent die Teilnahme an Veranstaltungen.

Dass Abgeordnete bei ihrer Arbeit vor Ort entgegen der häufig geäußerten Diagnose von der Entwicklung zur Mediendemokratie vorrangig auf die persönliche Kommunikation setzen, mag daran liegen, dass sie für ihr Alltagsgeschäft nur schwer Beachtung finden. Allerdings hängen die Erfolgsaussichten deutlich von der Situation im Wahlkreis ab. So berichtet ein FDP-Abgeordneter aus dem Allgäu:

38 Vgl. etwa Otfried Jarren / Patrick Donges, Politische Kommunikation in der Mediengesellschaft. Eine Einführung, Wiesbaden 2006.

39 Vgl. Stefan Marschall, Medialisierung komplexer politischer Akteure - Indikatoren und Hypothesen am Beispiel von Parlamenten, in: Frank Marcinkowski / Barbara Pfetsch (Hrsg.), Politik in der Mediendemokratie, Wiesbaden 2009, S. 205 - 223, S. 219.

$40 \mathrm{Vgl}$. Annette Knaut, Abgeordnete als Politikvermittler. Zum Wandel von Repräsentation in modernen Demokratien, Baden-Baden 2009, S. 238 f.

41 Frage im Beobachtungsbogen: „War die Veranstaltung erkennbar medienöffentlich, das heißt, waren Medienvertreter erkennbar anwesend?“" Ja: 453, Nein: 165. 


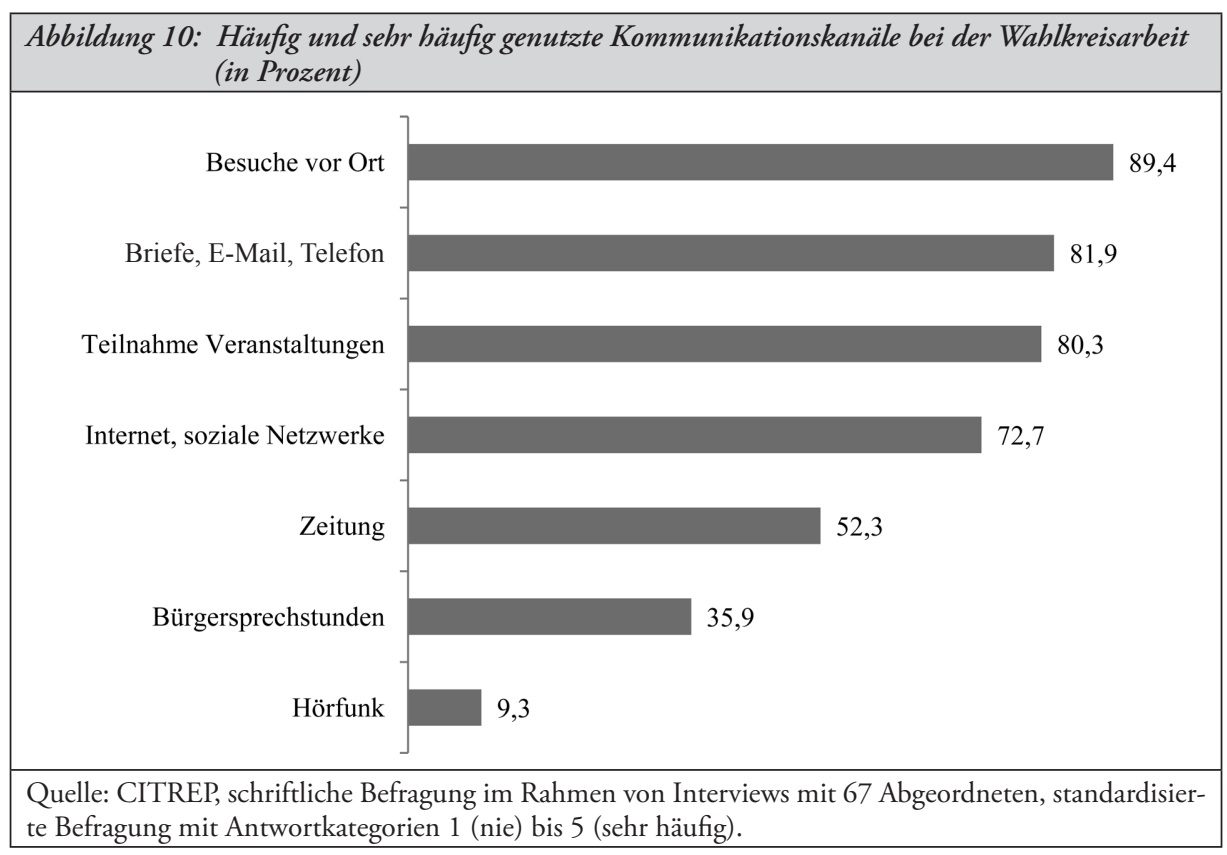

„Weiterhin versuche ich auch über alle Termine und Veranstaltungen, die es hergeben, eine kurze Pressemitteilung von zwanzig Zeilen in die Zeitung zu bringen. Die Zeitungen veröffentlichen [davon] in der Regel neun von zehn ... Gelegentlich kommt mal eine nicht, aber im Wesentlichen wird das dann auch gedruckt." (Stephan Thomae, MdB, FDP)

Hingegen kritisiert ein CDU-Abgeordneter aus Baden-Württemberg:

„Im Prinzip ist die Zeitung der zentrale Punkt, aber es ist sehr schwer, in der Zeitung genannt zu werden. Ich bin damit nicht zufrieden, das sage ich ganz offen. Die Zeitungen haben meines Erachtens ganz klar einen Auftrag, die Menschen zu informieren. Dem kommen sie nur sehr unzureichend nach. Auch bei den lokalen Zeitungen ist der Eventcharakter sehr, sehr hoch und der Informationsgehalt oftmals gering. Das hat sich in den letzten Jahren verschlechtert." (Josef Rief, MdB, CDU)

Verallgemeinern kann man dies nicht. Beim Blick auf die Zusammenhänge mit anderen Variablen wird deutlich, dass weder die Größe der Partei noch die geographische Lage des Wahlkreises einen wesentlichen Einfluss auf die Nutzung von Zeitungen zur Kommunikation hat. Allenfalls wird deutlich, dass in ländlichen Wahlkreisen der Gebrauch von Printmedien als Kommunikationsweg etwas höher ist (56 Prozent der Abgeordneten dort geben häufig oder sehr häufig an) als in städtischen (44 Prozent). Die Chancen der Abgeordneten, für ihre Wahlkreisarbeit publizistische Beachtung zu finden, sind in weiteren Analysen vertieft zu untersuchen. Neben den Unterschieden von Strukturmerkmalen im Wahlkreis mag hier insbesondere der rückläufige publizistische Wettbewerb als Folge der Pressekonzentration eine Rolle spielen ${ }^{42}$, aber auch die individuelle Professionalität der Pressearbeit durch

42 Vgl. Horst Röper, Zeitungsmarkt 2012: Konzentration erreicht Höchstwert. Daten zur Konzentration der Tagespresse in der Bundesrepublik Deutschland im I. Quartal 2012, in: MediaPerspektiven (2012), H. 5, S. $268-285$. 
die Abgeordnetenbüros. Dies näher zu ergründen, ist eine für die Praxis parlamentarischer Repräsentation höchst wichtige Aufgabe.

Angesichts des begrenzten Zugangs zur Medienberichterstattung werden in die Möglichkeiten des Internets und der neuen sozialen Netzwerke hohe Erwartungen für die direkte Kommunikation von Repräsentanten und Repräsentierten gesetzt. ${ }^{43}$ In einer vergleichenden Untersuchung der Homepage-Nutzung durch Parlamentarier in Deutschland, Schweden und den USA kommt Thomas Zittel allerdings zu einer abwartenden Einschätzung, sieht aber bei den Abgeordneten des Bundestages Anzeichen für Entwicklungen, die auf einen „Strukturwandel in der Wählerkommunikation“ hindeuten und letztlich einen „Demokratiewandel“ bewirken könnten; um hierzu weiteren Aufschluss zu erhalten, bedürfe es aber näherer Untersuchungen des Zusammenhanges mit den klassischen Formen der Kommunikation im Wahlkreis. ${ }^{44}$ Inzwischen ist die Nutzung des Internets als ein fester Kanal für die Kommunikation der Abgeordneten etabliert: 73 Prozent geben an, es häufig oder sehr häufig zu nutzen (vgl. Abbildung 10). Einige Abgeordnete gehen sogar weiter: „Mittlerweile ist das Internet das wichtigste" Medium (Petra Sitte, MdB, Die Linke), und ein CDU-Abgeordneter formuliert:

„Da die Zeitungen, die zwei großen Stuttgarter Zeitungen, schwierig sind, was die Kommunikation der Wahlkreisarbeit und der Bundestagstätigkeit angeht, sind schon eher soziale Medien wie Facebook, Briefe aus Berlin, eigene E-Mail-Verteiler und die Lokalzeitungen beziehungsweise Lokalteile der großen Zeitungen die Hauptwege der Kommunikation." (Stefan Kaufmann, MdB, CDU)

Besonders herausgestellt wird von vielen Abgeordneten die Bedeutung des sozialen Netzwerkes Facebook. 27 der 67 Interviewten (40 Prozent) machen hierzu nähere Ausführungen und erläutern, wie sie es für die wahlkreisbezogene Kommunikation einsetzen:

„Was ich jetzt feststelle, was sich immer mehr intensiviert, ist Facebook. Es kommen immer mehr Leute, auch aus meinem Wahlkreis, die sagen, ich möchte befreundet sein, und dann auch posten." (Petra Ernstberger, MdB, SPD)

„Ein Großteil der Korrespondenz läuft ja über Facebook, was ich inzwischen auch lernen musste, über E-Mails, aber auch anhand von Briefen." (Hans-Christian Ströbele, MdB, Bündnis 90/Die Grünen)

Der Kurznachrichtendienst Twitter hingegen wird nur von zehn Abgeordneten (15 Prozent) genannt, neun berichten von ihrem Umgang mit dem Online-Portal Abgeordnetenwatch (dreizehn Prozent), nur vier von eigenen Blogs (sechs Prozent). Dies findet eine klare Entsprechung im Rezeptionsverhalten der Bevölkerung: In der im Rahmen der Untersuchung durchgeführten repräsentativen Befragung antworten auf die Frage, ob sie sich über Politiker und deren Arbeit mittels Twitter informieren, 89 Prozent mit „niemals“ (zu Facebook: 85

43 Vgl. etwa Dorothea Lamatsch / Alexander Bilgeri, Der MdB im WWW, in: Alexander Siedschlag (Hrsg.), Kursbuch Internet und Politik. Schwerpunkt: Wahlkampf im Netz, Opladen 2002, S. 75 - 90; Annette Knaut, Politikvermittlung online: Abgeordnete des Deutschen Bundestages im Web 2.0, in: Gerhard Göhler / dies. (Hrsg.), Medien - Demokratie, Frankfurt am Main 2010, S. 9 - 37; im Überblick: Jürgen Stern, Web 2.0 trifft Politik 3.11: Bringt politische Kommunikation durch das Internet mehr Transparenz, Partizipation und Legitimität?, in: Werner J. Patzelt / Martin Sebaldt / Uwe Kranenpohl (Hrsg.), Res publica semper reformanda. Wissenschaft und politische Bildung im Dienste des Gemeinwohls, Wiesbaden 2007, S. 168 - 179.

44 Vgl. Thomas Zittel, Die elektronische Wählerkommunikation von Abgeordneten aus vergleichender Perspektive - Medienwandel oder Demokratiewandel?, in: ZPol, 18. Jg. (2008), H. 2, S. 185 -208 , S. 204. 
Prozent) und sieben Prozent mit „selten“ (Facebook: neun Prozent). ${ }^{45}$ Die sozialen Medien mögen mittlerweile also fest etablierte Werkzeuge aus dem „Instrumentenkasten“ der Abgeordnetenkommunikation sein, doch große direkte Wirkung können sie kaum entfalten und substituieren die alten Medien und die direkte Kommunikation bislang nicht. Dies entspricht auch den Ergebnissen von Untersuchungen zu deren häufig überschätzter Rolle in Wahlkämpfen ${ }^{46}$. Vermutlich spielen sie vor allem eine Rolle beim Austausch mit Journalisten, aktiven Parteimitgliedern und sonstigen „Political Junkies“; insofern sind sie als Teil des Zwei-Stufen-Flusses der politischen Kommunikation (Paul F. Lazarsfeld) ${ }^{47}$, in dem solchen Meinungsbildnern eine wichtige Rolle zukommt, doch nicht gänzlich zu vernachlässigen.

Insgesamt kann also festgehalten werden, dass bei allem Wandel in den technischen Möglichkeiten des Internets die Abgeordneten ihre Repräsentationsfunktion vor Ort in den Wahlkreisen weiterhin auf geradezu klassische Art und Weise hauptsächlich von Angesicht zu Angesicht persönlich wahrnehmen, während die neuen und alten Medien allenfalls ergänzend, nicht aber substituierend wirken. Das für die Arbeit in der Hauptstadt verschiedentlich aufgezeigte enge Verhältnis von Politik und Medien, das teils symbiotische Züge annimmt ${ }^{48}$, ist im Wahlkreis offenbar deutlich schwächer ausgeprägt.

\section{Die Wahlkreisarbeit der Abgeordneten: unverzichtbar für demokratische Repräsentation}

Die Wahlkreisarbeit der Abgeordneten des Deutschen Bundestages hat zwar bisher in der Forschung wenig Beachtung gefunden. Für das Funktionieren wie das Verständnis des parlamentarischen Systems ist sie aber unverzichtbar. Abgeordnete verbringen vor Ort auch außerhalb von Wahlkämpfen regelmäßig viel Zeit, richten ein breites Kontaktangebot an die Bevölkerung und pflegen die Verbindung zu ihrer lokalen Parteiorganisation. Sie entsprechen damit den Erwartungen, die aus den institutionellen Anreizen des Wahl- und insbesondere des Nominierungssystems in Deutschland resultieren. Ein systematischer Unterschied im Verhalten von über Landeslisten und direkt gewählten Abgeordneten fällt in deren Repräsentationsverhalten indes nicht auf: Beide tun im Prinzip dasselbe. Deutlich im Vordergrund der Wahlkreisarbeit steht die Informationsaufnahme im Rahmen von Kontakten mit öffentlichen Einrichtungen und Privatunternehmen, mit Vereinen und anderen Organisationen. Darüber hinaus bieten die Abgeordneten vielfach Hilfestellungen und Dienstleistungen im Einzelfall an.

Die Wahlkreisarbeit weist vier funktionale Dimensionen auf: Wahl, Nominierung, Information und Kommunikation. Mit ihrer Hilfe lässt sich die Ausgestaltung der Wahlkreisarbeit

45 CITREP Bevölkerungsbefragung, 2010, 1.553 Befragte, Antworten zur vorliegenden Frage $\mathrm{N}=1.016$.

46 Vgl. Thorsten Faas / Ansgar Wolsing / Sascha Huber, Die überschätzte Gefahr: Beeinflussen (getwitterte) Umfrageergebnisse Wahlentscheidungen?, in: Evelyn Bytzek / Sigrid Roßteutscher (Hrsg.), Der unbekannte Wähler? Mythen und Fakten über das Wahlverhalten der Deutschen, Frankfurt am Main 2011, S. 177 - 191, S. 185.

47 Vgl. Paul F. Lazarsfeld / Bernard Berelson / Hazel Gaudet, The People's Choice. How the Voter Makes up his Mind in a Presidential Campaign, New York 1944, S. 151.

48 Vgl. Philip Baugut / Maria-Theresa Grundler, Politische (Nicht-)Öffentlichkeit in der Mediendemokratie. Eine Analyse der Beziehungen zwischen Politikern und Journalisten in Berlin, BadenBaden 2010; Ulrich von Alemann, Parteien und Medien, in: Oscar W. Gabriel / Oskar Niedermayer I Richard Stöss (Hrsg.), Parteiendemokratie in Deutschland, Opladen 2002, S. 467 - 483. 
gut erklären und aufzeigen, dass sich die Abgeordneten im Großen und Ganzen entsprechend der daraus resultierenden Anreize verhalten: Bürgerorientierung, Parteipflege, Informationsaufnahme und persönliche Kommunikationsangebote werden dort groß geschrieben.

Vielfältige Fragestellungen, die hier teilweise schon angesprochen wurden, sind in weiteren Analysen zu vertiefen: In welchem Zusammenhang stehen die Abgeordnetenarbeit und das Vertrauen der Bevölkerung in die Demokratie? ${ }^{49}$ Welche Ansprüche stellen die Bürger an die Repräsentation, und wie bewerten sie die Praxis? ${ }^{50}$ Wie sind Wahlkreis- und Parteiarbeit miteinander verschränkt? ${ }^{51}$ Wie wirken institutionelle Anreize auf das Abgeordnetenhandeln in unterschiedlichen politischen Systemen?52 Inwieweit und wann übernehmen Parlamentarier im Wahlkreis politische Führungsfunktionen? (Wie) kann das Rollenverhalten im Wahlkreis gruppiert werden - und wovon hängt eine Zuordnung ab? ${ }^{53}$ Gibt es für MdBs einen Hauptstadt- und einen Wahlkreisstil in ihrem persönlichen Auftreten? Lassen sich bei weiterer Betrachtung doch Unterschiede zwischen Inhabern von Listen- und Direktmandaten erkennen? Wie werden Abgeordnete in ihrer Wahlkreisarbeit sozialisiert? Was sind die Stärken und Gefahren der Hilfeleistungen für Bürger im Einzelfall? Wie kooperieren Parlamentarier unterschiedlicher Ebenen im föderalen System der Bundesrepublik miteinander?

In der politischen Praxis haben Abgeordnete immer wieder erfahren, dass sie ihre Wahlkreise und die Arbeit dort nicht vernachlässigen sollten. Gleiches gilt für politische und wissenschaftliche Beobachter, wollen sie sich ein realistisches Bild von der Abgeordnetentätigkeit und den Mechanismen moderner repräsentativer Demokratien verschaffen: Bei allen Möglichkeiten der Informationsaufnahme aus Umfrageforschung und Medienberichterstattung sowie bei allen technischen Lösungen für die gezielte Wählerkommunikation über E-Mail, Websites und soziale Netzwerke wie Facebook oder Twitter - im Alltag ist das Geschäft der demokratischen Repräsentation weiterhin vor allem geprägt von der persönlichen Diskussion, dem Austausch von Meinungen, der Aufnahme von Informationen im Gespräch. Dies ist beruhigend angesichts von Szenarien technokratischer Regierungssysteme der "Postdemokratie“, in denen Input nur noch aus der Demoskopie aufgenommen, Entscheidungen im Spiel von Lobbyisten und Politikberatern getroffen und Output nur noch über die Massenmedien vermittelt wird. ${ }^{54}$

Ob die Abgeordneten bei ihrer Wahlkreisarbeit die rechte Balance finden zwischen der Aufnahme und der Vermittlung von Politik, zwischen der Hilfe im Einzelfall und der Berücksichtigung von Gruppeninteressen, zwischen Partei- und Bürgerkontakten, zwischen Ignoranz und Nutzung der Medienlogik, zwischen dem Einsatz neuer und alter Medien das alles kann weder empirisch noch normativ abschließend wissenschaftlich festgestellt werden. Es lohnt aber für jeden Praktiker und jeden Parlamentsforscher, dies näher zu prüfen und immer wieder zu diskutieren. Für politisch interessierte Menschen jedenfalls, das ist im Rahmen der Beobachtungen deutlich geworden, besteht jederzeit die Möglichkeit, ihre Abgeordneten im Wahlkreis zu kontaktieren, um über brennende - oder alltägliche - Fragen zu sprechen. Auch diese Holschuld der Bevölkerung gilt es zu beachten.

49 Vgl. den Beitrag von Oscar W. Gabriel in diesem Heft der ZParl.

50 Vgl. den Beitrag von Mirjam Dageförde in diesem Heft der ZParl.

51 Vgl. den Beitrag von Danny Schindler in diesem Heft der ZParl.

52 Vgl. Olivier Costa / Sven T. Siefken, MPs Working in their District in France and Germany, Vortrag im Rahmen der DVParl-Konferenz „Repräsentation im Wahlkreis - Bevölkerung und Abgeordnete in Deutschland und Frankreich“, Berlin am 7./8. Dezember 2012.

53 Vgl. Sven T. Siefken, a.a.O. (Fn. 35).

54 Vgl. Colin Crouch, Postdemokratie, Frankfurt am Main 2008, S. 10 f., S. 99. 